\title{
What factors must be considered in 'return to school' following concussion and what strategies or accommodations should be followed? A systematic review
}

\author{
Laura K Purcell, ${ }^{1}$ Gavin A Davis, ${ }^{2}$ Gerard A Gioia ${ }^{3}$
}

\begin{abstract}
- Additional material is published online only. To view please visit the journal online (http://dx.doi.org/10.1136/ bjsports-2017-097853).

1 Department of Pediatrics, McMaster University, Hamilton, Ontario, Canada

${ }^{2}$ Murdoch Children's Research Institute, Melbourne, Victoria, Australia

${ }^{3}$ Division of Pediatric Neuropsychology, Children's National Health System, Rockville, Maryland, USA
\end{abstract}

Correspondence to Dr Laura K Purcell, Department of Pediatrics, McMaster University, Hamilton, ON L8S 4L8, Canada;

Ipurcell1015@rogers.com

This study was presented in part at the 5th International Consensus Conference on Concussion in Sport in Berlin 2016, and some of these results were published with the conference proceedings.

Received 28 March 2017 Revised 8 January 2018 Accepted 2 February 2018 Published Online First 2 March 2018

Check for updates

To cite: Purcell LK, Davis GA Gioia GA. Br J Sports Med 2019:53:250.

\author{
ABSTRACT \\ Objective To evaluate the evidence regarding (1) \\ factors affecting return to school (RTS) and (2) strategies/ \\ accommodations for RTS following a sport-related \\ concussion (SRC) in children and adolescents. \\ Design A systematic review of original studies \\ specifically addressing RTS following concussion in the \\ paediatric and sporting context.
}

Data sources MEDLINE (Ovid), Embase (Ovid), Psyclnfo (Ovid) electronic databases and the grey literature OpenGrey, ClinicalTrials.gov and Google Advanced. Eligibility criteria Studies were included if they were original research on RTS following SRC in children aged 5-18 years published in English between 1985 and 2017.

Results A total of 180 articles were identified; 17 articles met inclusion criteria. Several factors should be considered for RTS after concussion, including: symptomatology; rest following injury; age/grade; and course load. On RTS after concussion, $17 \%-73 \%$ of students were provided academic accommodations or experienced difficulty with RTS. Students were more likely to obtain academic accommodations in schools with a concussion policy if they had a medical RTS letter and had regular medical follow-up after concussion.

Conclusions Schools should have a concussion policy and offer individualised academic accommodations to students recovering from SRC on RTS; a medical letter should be provided to facilitate provision/receipt of academic accommodations; students should have early, regular medical follow-up following SRC to help with RTS and monitor recovery; students may require temporary absence from school after SRC; clinicians should assess risk factors/modifiers that may prolong recovery and require more intensive academic accommodations. PROSPERO registration number CRD42016039184.

\section{INTRODUCTION}

Concussions are common in sports, particularly in children and adolescents, with about $70 \%$ of sport-related head injuries occurring in persons under 18 years of age. ${ }^{1}$ About 100000-175000 children present annually to US emergency departments (ED) with sport-related concussion (SRC), which accounts for $25 \%-50 \%$ of paediatric concussions. $^{2-5}$ Concussions represent $9 \%-12 \%$ of sport injuries in high school athletics. ${ }^{67}$

Children face different issues than adults after SRC, with RTS being a key aspect of management. For children and adolescents who recover quickly from SRC, school return may require minimal support. However, postconcussion cognitive symptoms such as impaired memory, attention and concentration, and somatic symptoms such as headaches, dizziness and fatigue may negatively impact students' ability to RTS. ${ }^{8-10}$ Students with more numerous/severe symptoms may have symptom exacerbation with RTS. ${ }^{11}$

Several consensus/position statements and guidelines have addressed RTS after concussion. ${ }^{12-16}$ Following the Fourth International Consensus Conference on Concussion in Sport, the ChildSCAT3 assessment tool was developed for children aged 5-12 years, with a child-specific symptom scale and recommendations for RTS. The Concussion in Sport Consensus Statement also addressed children's cognitive requirements and need for school accommodations. ${ }^{17}$ However, these resources have been based on limited empirical research. Many areas of RTS lack evidence-based guidelines.

Therefore, this systematic review addressed two questions:

1. What factors must be considered in 'return to school' following concussion?

2. What strategies or accommodations should be recommended in 'return to school' following concussion?

\section{METHODS}

This systematic review was conducted as part of a larger review on differences in concussion management between children and adults, which was presented at the Fifth International Consensus Conference on Concussion in Sport. ${ }^{18-20}$ However, it represents a more detailed analysis of RTS in children and adolescents following SRC.

This systematic review was prospectively registered in the PROSPERO database (registration number CRD42016039184).

\section{Literature identification}

The search strategy (box 1) was developed in collaboration with a specialist librarian. Electronic databases searched were Ovid MEDLINE, Ovid Embase and Ovid PsycInfo, in addition to reviewing reference lists of retrieved articles and published articles by the authors to identify any potentially eligible articles not identified by the electronic database search. Three grey literature repositories (OpenGrey, ClinicalTrials.gov and Google Advanced) were searched. The searches addressing both 


\section{Box 1 Search strategy}

Ovid MEDLINE:

1. exp Brain Concussion/

2. concuss $\$$.tw.

3. ((mild or minor or minimal) adj (traumatic brain or tbi)).tw.

4. mtbi.tw

5. exp Brain Injury/

6. (((severe or moderate) adj2 (head or brain or traumatic or tbi)) not (mild or minor)).ti.

7. 5 not 6

8. or $/ 1-4,7$

9. 8 and (child* or adolescen*).mp.

10. 9 not (animal/not human/)

11. limit 10 to English

12. limit 11 to $\mathrm{yr}=$ "1985-Current"

13. (return* adj3 (school or learn*)).mp.

14. 12 and 13

Ovid Embase:

1. Brain Concussion/or Concussion/

2. concuss $\$ . t w$.

3. ((mild or minor or minimal) adj (traumatic brain or tbi)).tw.

4. mtbi.tw

5. exp Brain Injury/

6. (((severe or moderate) adj2 (head or brain or traumatic or tbi)) not (mild or minor)).ti.

7. 5 not 6

8. or $/ 1-4,7$

9. 8 and (child* or adolescen*).mp

10. 9 NOT ((exp animal/or nonhuman/NOT exp human)

11. limit 10 to English

12. limit 11 to $\mathrm{yr}=" 1985$-Current"

13. (return* adj3 (school or learn*)).mp.

14. 12 and 13

Ovid Psyclnfo:

1. exp Brain Concussion/

2. concuss $\$$.tw.

3. ((mild or minor or minimal) adj (traumatic brain or tbi)).tw.

4. mtbi.tw

5. exp Head Injuries/OR exp Traumatic Brain Injury/

6. (((severe or moderate) adj2 (head or brain or traumatic or tbi)) not (mild or minor)).ti.

7. 5 not 6

8. or $/ 1-4,7$

9. limit 8 to (childhood or adolescence $<13$ to 17 years $>$ )

10. (minors or minors* or boy or boys or boyfriend or boyhood or girl* or kid or kids or child or child* or children* or schoolchild* or schoolchild).mp. or school child.ti,ab. or school child*.ti,ab. or (adolescen* or juvenil* or youth* or teen ${ }^{*}$ or under ${ }^{*}$ age ${ }^{*}$ or pubescen*).mp. or exp pediatrics/or (pediatric* or paediatric* or peadiatric*).mp. or school.ti,ab. or school*.ti,ab.

11. 8 and 10

12. 9 or 11

13. limit 12 to English

14. limit 13 to $\mathrm{yr}=$ "1985-Current"

15. (return* adj3 (school or learn*)).mp.

16. 14 and 15 questions (factors and accommodations) were run concurrently; however, the results were analysed separately.

\section{Article selection}

Inclusion criteria were:

- original research on RTS following SRC

- in children aged 5 years to less than 18 years

- published in English

- between 1985 and May 2016 (results presented at the Berlin meeting).

The search was repeated for this updated review to include studies published between the initial search and June 2017.

Exclusion criteria were:

- studies of moderate/severe traumatic brain injuries

- patients with no clear history of head trauma or SRC

- patients who sustained non-accidental injury

- preschool-age children.

Studies evaluating patients with mixed mechanisms of injury were included if SRC was part of the study population. As well, studies with mixed age cohorts were included if there were sufficient numbers of children/adolescents less than 18 years of age. In addition, studies examining cognitive activity/rest or cognitive impairment following SRC were included as a proxy for school effects following concussion.

All titles and abstracts identified by the search were reviewed independently by two of the authors (LKP and GAD) and the full texts of all potentially eligible articles were obtained and reviewed. The final selection of articles was determined by consensus among the authors.

\section{Data extraction}

The following data from the included studies were inputted into a data extraction table independently by LKP and GAD: study design; participant characteristics; exposure/intervention; outcome; results; limitations.

\section{Risk of bias assessment}

Two authors (LKP and GAD) independently assessed the quality of each study using the Downs and Black (DB) checklist. $^{21}$ This tool can be used for randomised and non-randomised study designs. Discrepancies in DB scoring were resolved by consensus. The level of evidence for each study was assessed independently by each of the authors using The Oxford 2011 Levels of Evidence. ${ }^{22}$ All authors agreed on the assessed levels of evidence.

\section{RESULTS}

Seventeen studies were included (figure 1). ${ }^{23-39}$ The initial search generated 167 potentially eligible articles of which 11

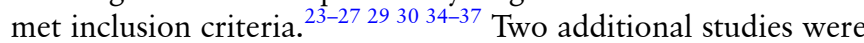
independently identified by one of the authors (GAG). ${ }^{38} 39$ The updated search identified 10 additional potential articles, of which three 283233 met inclusion criteria, and an additional article was identified by one of the authors (GAG). ${ }^{31}$ Many of the included studies evaluated mixed cohorts of children, adolescents and adults, as well as varied mechanisms of injury not related to sport. Frequent areas of limitations for the included studies included: lack of reported adverse events; external validity for most studies as the populations studied were recruited from urban EDs or specialty concussion clinics; lack of blinding and randomisation. In addition, the overall level of evidence was low (3 or 4); there were only two randomised controlled trials (RCT) identified. $^{36} 38$ 


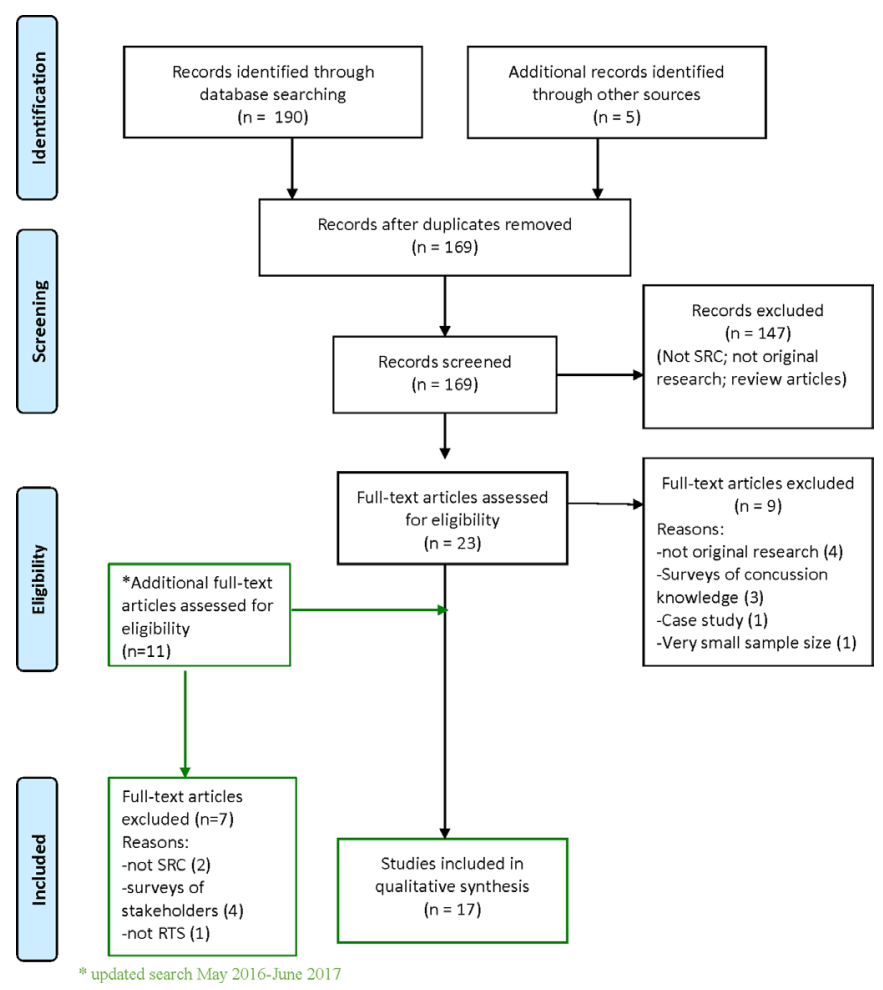

Figure 1 Preferred Reporting Items for Systematic Reviews and MetaAnalyses (PRISMA) flow diagram. RTS, return to school; SRC, sportrelated concussion.

\section{Factors to be considered on RTS following concussion}

Eleven articles examined factors to be considered after concussion on RTS (table 1). ${ }^{23-33}$ These factors include:

1. Symptom load/severity: Higher symptom loads and more severe symptoms were associated with a greater likelihood of missing more days of school and having difficulties on RTS, in addition to longer recovery. ${ }^{23} 2529-31$

2. Types of symptoms: Symptoms such as headache, visual disturbances, memory deficits, difficulty concentrating, executive dysfunction and vestibular abnormalities may adversely affect a student's ability to attend school after a concussion. $^{2325-272931}$

3. Duration of symptoms: Patients with longer duration of symptoms had more difficulty with RTS $^{23}{ }^{30}$; high cognitive loads after injury were associated with persistent symptoms. ${ }^{24}$

4. Age/grade or school level: Adolescents/high school students tended to have more postconcussion symptoms, greater severity of symptoms, and took longer to recover, to RTS and to return to play (RTP) than younger children. ${ }^{25}{ }^{29-31}$ In addition, adolescents were more concerned about negative academic effects of concussion than younger children. ${ }^{30}$

5. Course load: Certain subjects such as math pose greater problems for students on RTS after a concussion, followed by reading/language, arts, science and social studies. ${ }^{30}$

6. Rest following injury: Patients who did not initially rest following injury took longer to RTS than those who rested immediately following injury ${ }^{32}$; high cognitive loads/increased school attendance exacerbated symptoms. ${ }^{24} 28$

Socioeconomic status (SES) may also affect concussion recovery. A US pilot study examining SES and effect on concussion recovery found that patients with private insurance missed more days of school following concussion than patients with public insurance (mean of 5.2 days \pm 10.6 vs 2.0 days \pm 2.1 ) ${ }^{33}$
Additional factors to be considered include school concussion policies/resources, medical guidance to schools following concussion diagnosis and medical follow-up after concussion. ${ }^{36} 3739$

\section{Strategies or accommodations for RTS}

Six articles examined strategies or provision/receipt of accommodations for RTS (table 2). ${ }^{34-39}$ Other than temporary school absence, the types of academic accommodations provided were not specified. Most students were able to RTS after 2-5 days, ${ }^{293738}$ although up to $45 \%$ of students may experience exacerbation/ recurrence of symptoms on RTS. ${ }^{34}$ On RTS after concussion, $17 \%-73 \%$ of students were provided academic accommodations and/or experienced school difficulty. ${ }^{23252629353739}$ An RCT found that academic accommodations were more likely to be initiated for students recovering from concussion in schools with concussion policies. ${ }^{36}$ Concussed students who received outpatient medical follow-up, parental education and a medical RTS letter after initial assessment in an ED were also more likely to receive academic accommodations on RTS. ${ }^{37} 39$ Some academic subjects, such as mathematics and reading/language, may pose greater problems for students on RTS following a concussion, suggesting that these subjects might require more intervention. $^{30}$

\section{DISCUSSION}

\section{Factors to be considered on RTS following concussion}

Although historically concussion management has focused on return to sport, RTS is more important in children and adolescents, since school is their primary 'work'. ${ }^{40}$ Four factors should be considered on RTS after concussion ${ }^{23-33363739}{ }^{\text {: }}$

1. symptomatology

2. age/school level

3. course load

4. rest following injury.

Students with more symptoms, greater symptom severity, more persistent symptoms and particular symptoms such as difficulty concentrating, executive dysfunction, visual deficits and vestibular abnormalities took longer to recover and had more difficulty with RTS. ${ }^{232526-3135}$ Visual abnormalities and vestibulo-ocular abnormalities are particularly common after concussion and in patients with persistent symptoms. ${ }^{41-45}$

Adolescents/high school students tended to have more symptoms, more severe symptoms and more difficulty with RTS than younger students, and took longer to recover. ${ }^{29} 303439$ Age 13 years old and greater was a predictor of persistent concussion symptoms in a recent prospective, multicentre ED cohort study. ${ }^{46}$ The reasons for age and school level differences are unclear but may reflect the nature of injury manifestation in adolescents; increased academic and social demands; greater challenges to implement consistent, coordinated management strategies across multiple classes/teachers; and greater independence and decreased supervision in compliance with medical recommendations. These studies, however, sampled children and adolescents from specialty clinics, which may represent an overall higher symptom burden and severity, and therefore may limit the generalisation of their findings.

Lack of rest, both cognitive and physical, immediately following concussion has also been associated with persistent symptoms. Higher cognitive activity, such as school attendance, may cause exacerbation of symptoms and prolonged recovery from concussion, as well as longer RTS. ${ }^{11242832}$ Student-athletes who continued to play after injury took twice as long to recover and were almost nine times as likely to have prolonged recovery 


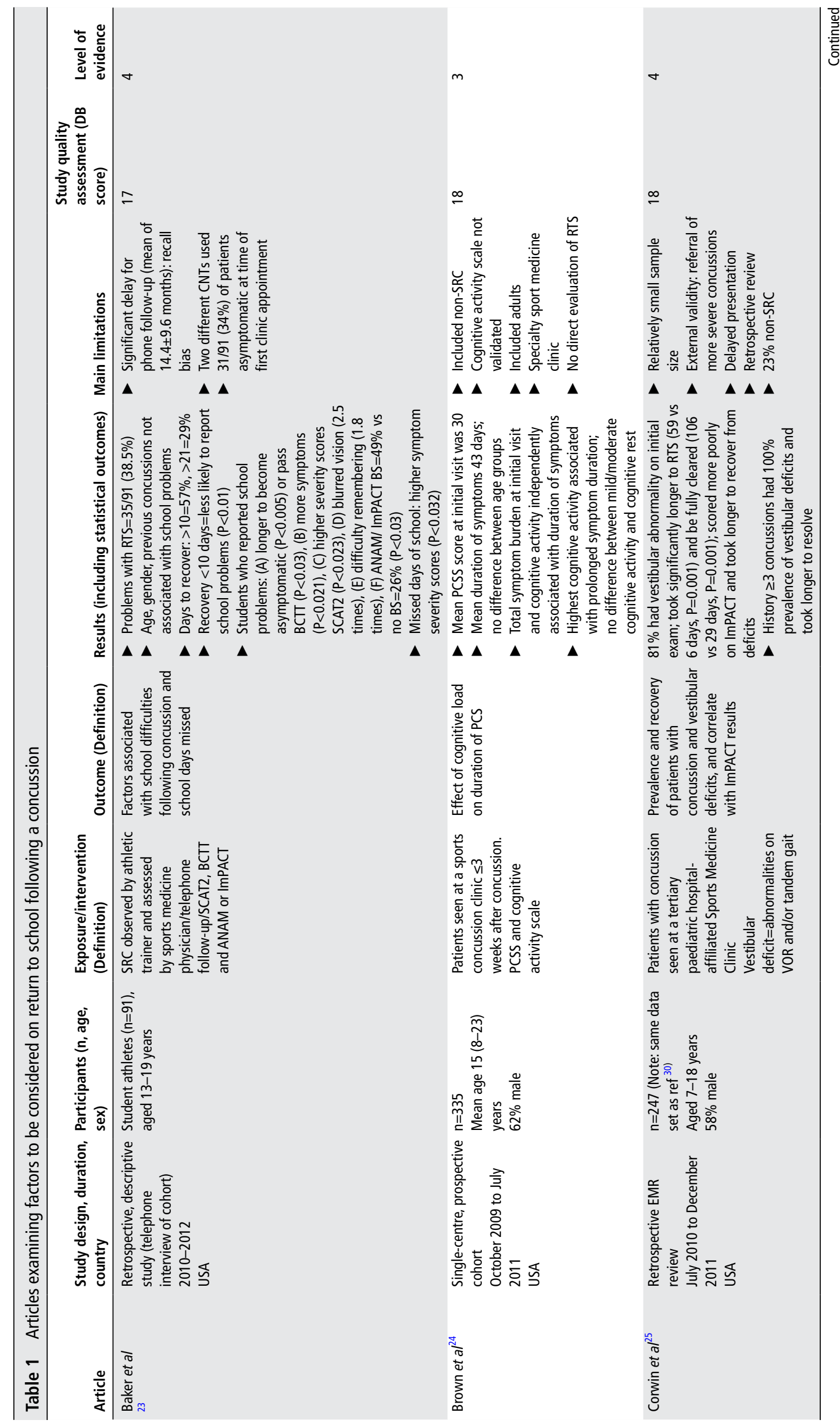




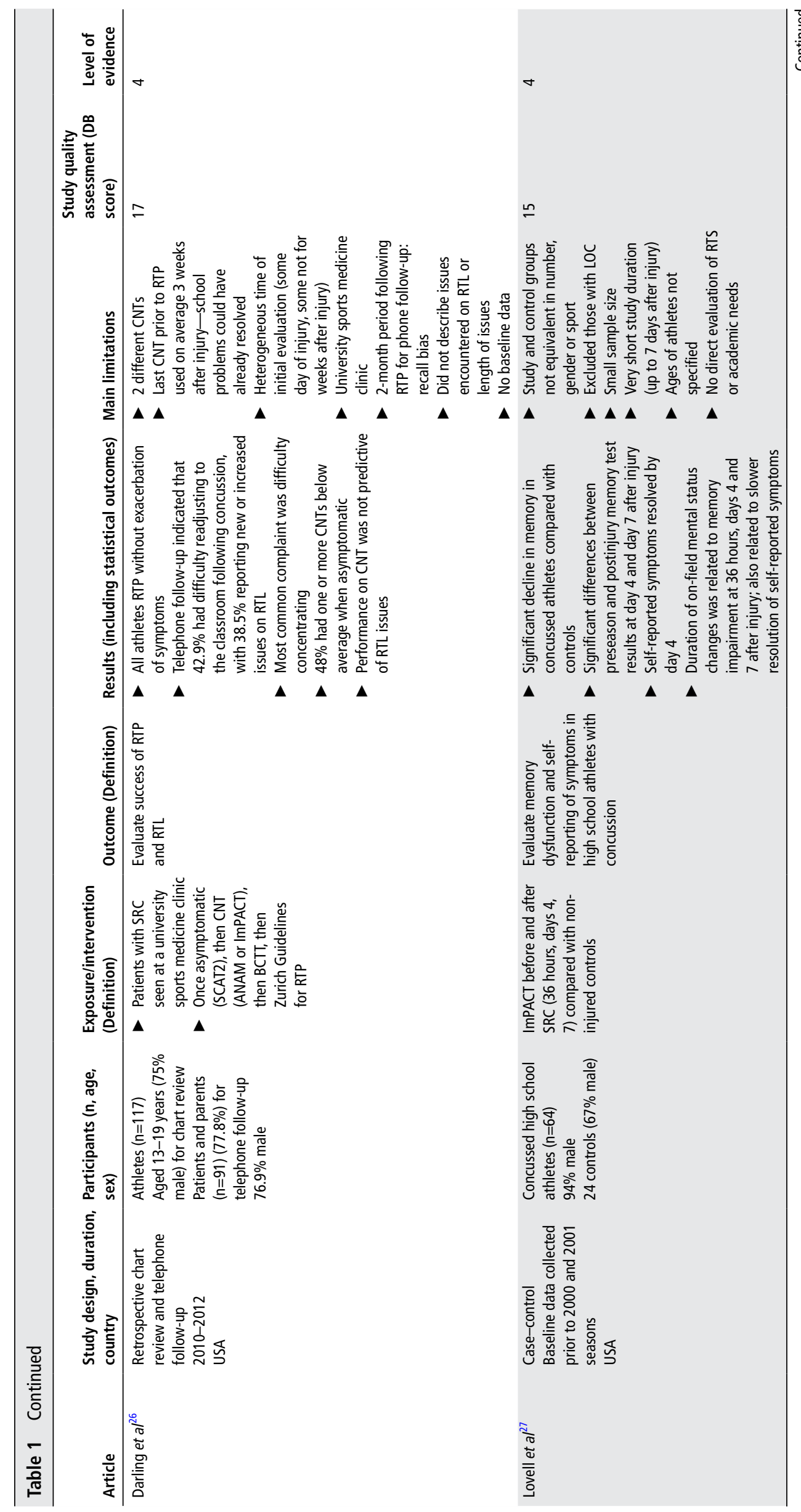

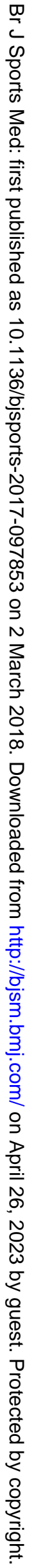




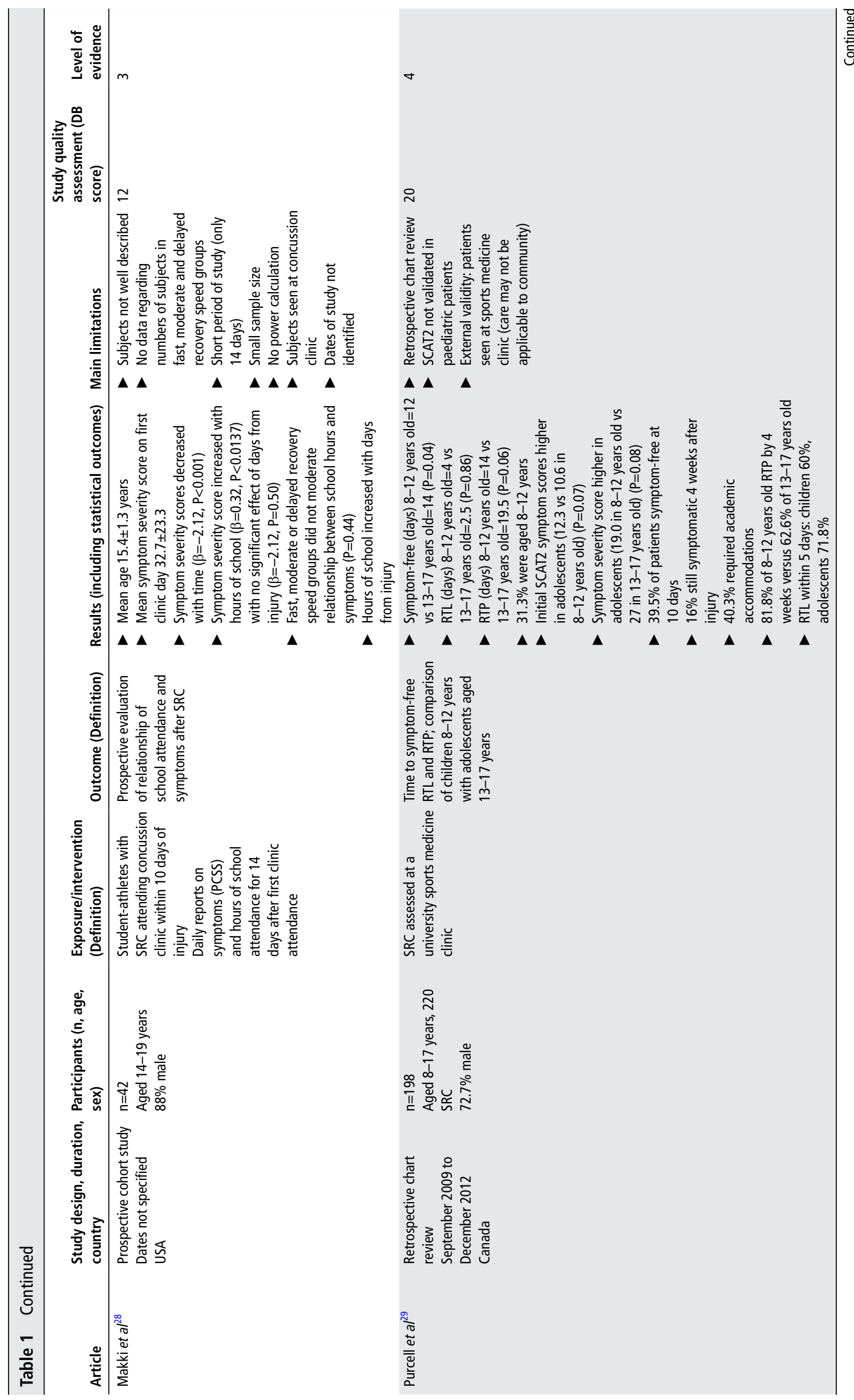




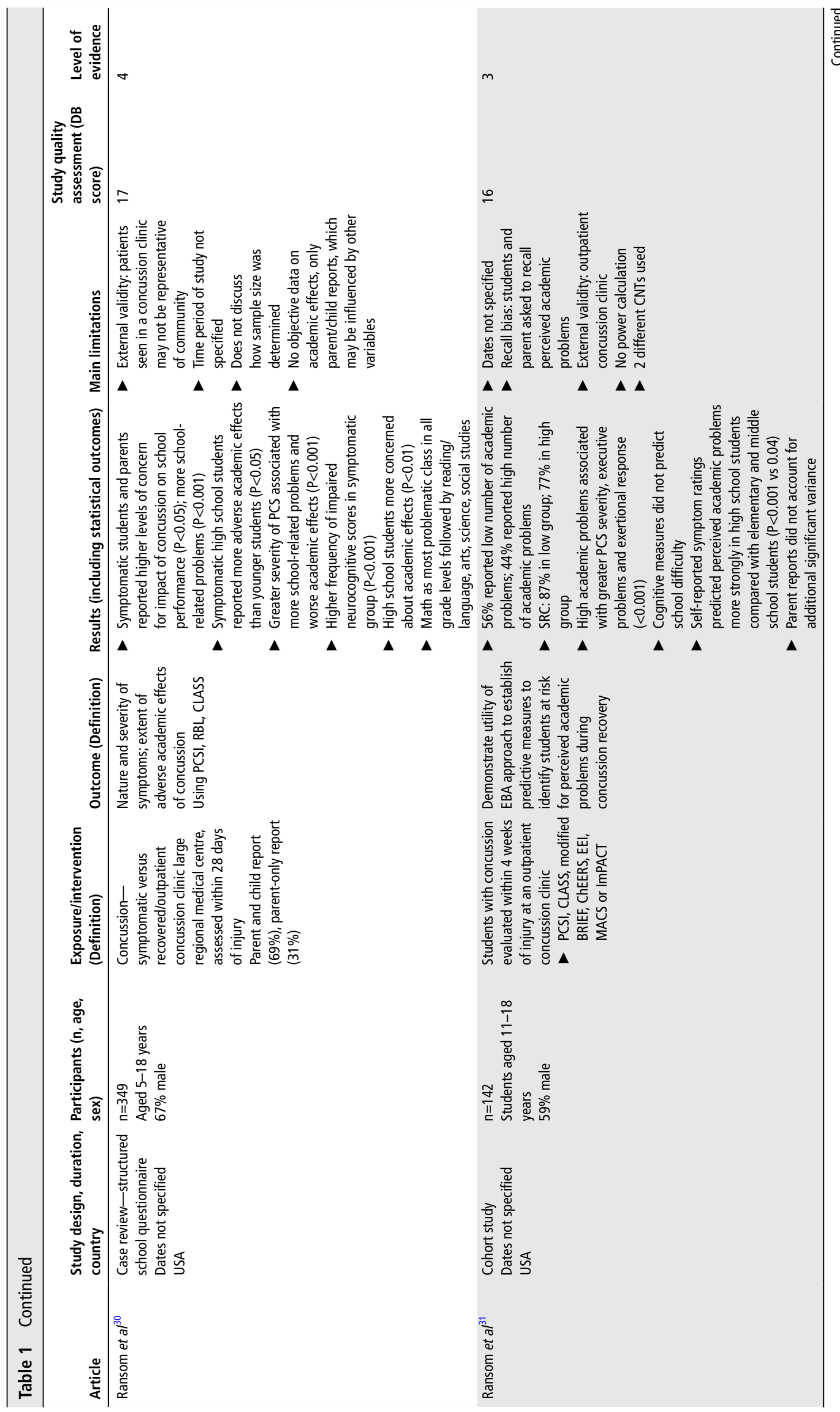




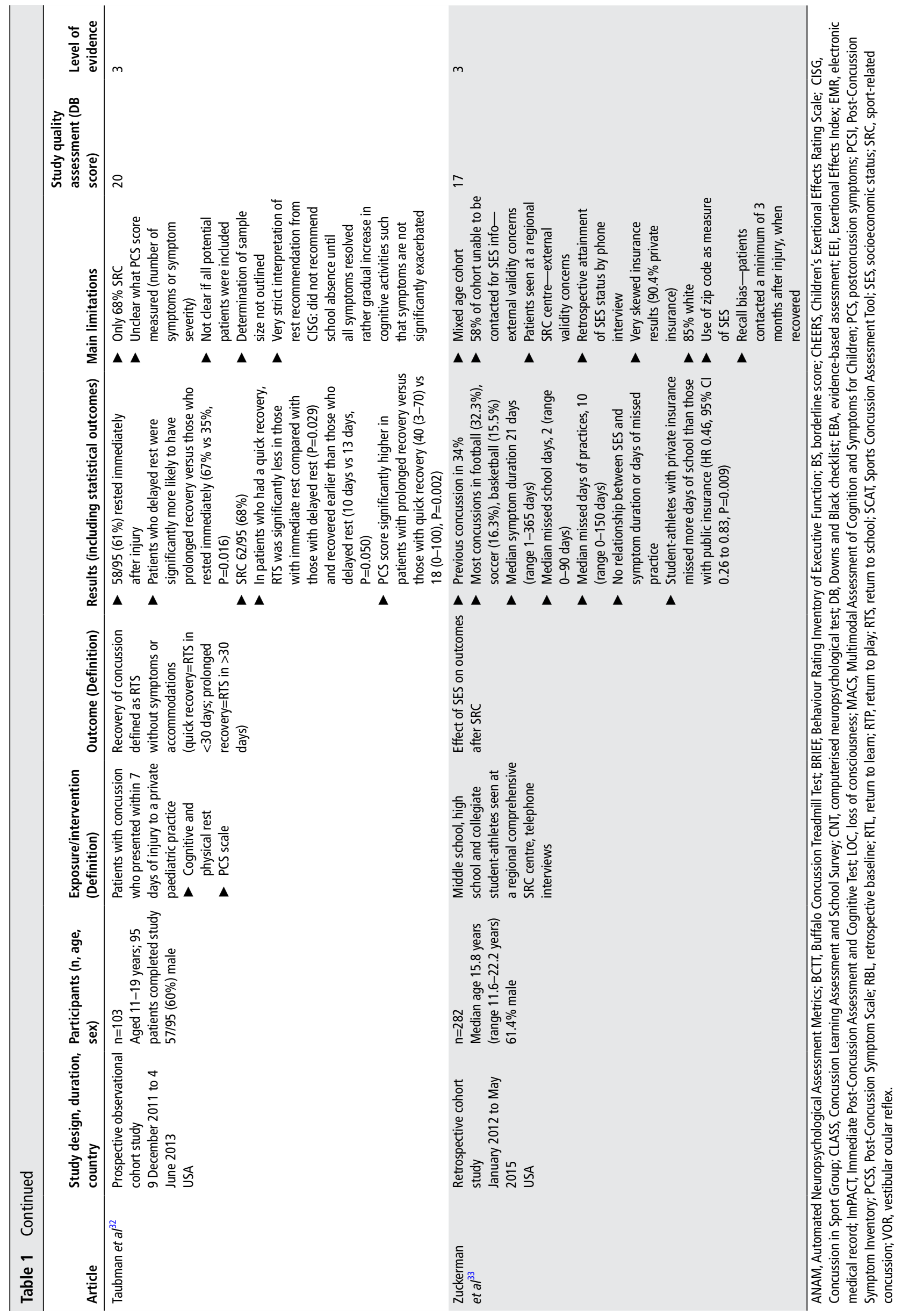




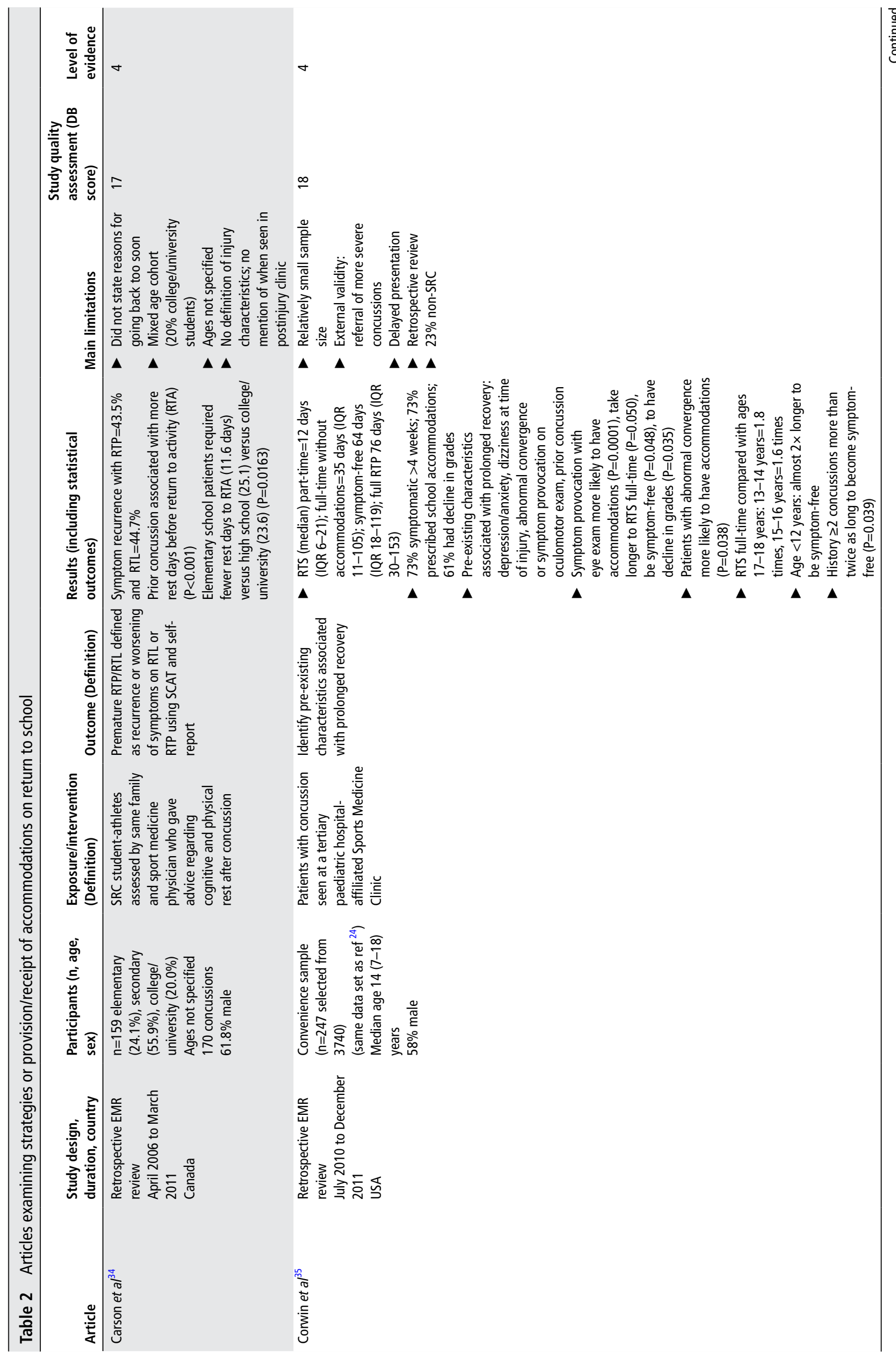




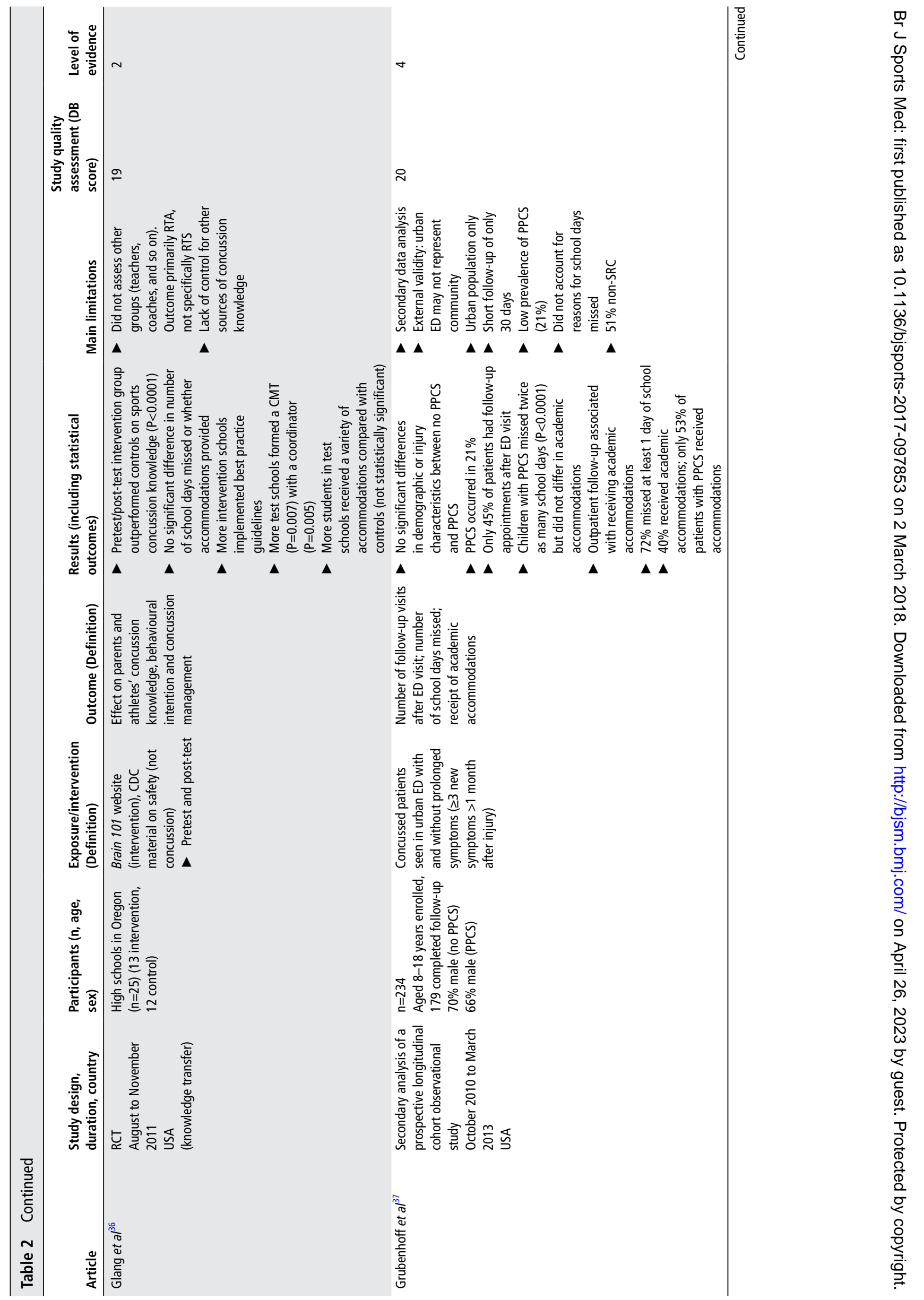




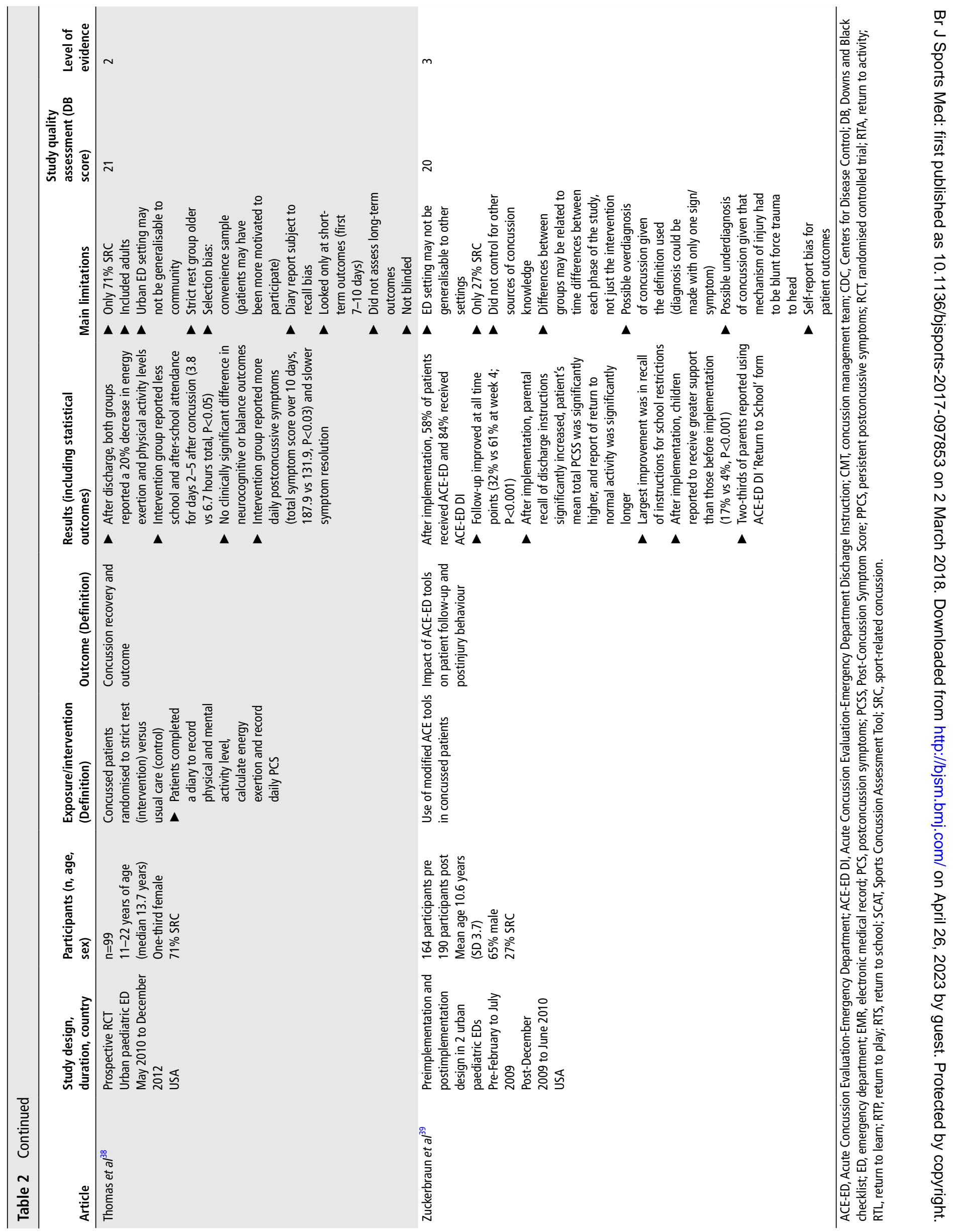


(>21 days) compared with those who were removed from play immediately after injury. ${ }^{47}$ Although RTS was not specifically assessed, cognitive performance was lower and symptoms higher in adolescents not removed from play immediately after concussion. ${ }^{47}$ The presence of risk factors/predictors of persistent symptoms should prompt practitioners to be more cognisant of potential difficulties in RTS and the need for more support in the RTS process.

An additional factor possibly affecting RTS after concussion is SES, though it requires more detailed study to fully understand its significance. The type of family insurance (private vs public), as a proxy for SES, may impact length of school absence following concussive injury, with concussed student-athletes with private insurance missing more days of school than those with public insurance. ${ }^{33}$ The reasons for this difference in school attendance were not identified; however, the study population represented only about $40 \%$ of the cohort and $90 \%$ had private insurance, raising concerns about external validity and selection bias. As well, five other markers of SES were not associated with differences in recovery, which makes the significance of SES in concussion recovery unclear.

Very likely there are other factors and specific deficits after concussion, such as autonomic dysfunction, that may impact on RTS that have not yet been studied in that context.

\section{Strategies or accommodations for RTS}

Significant numbers of symptomatic students (17\%-73\%) may require academic accommodations on RTS after concussion $^{29353739}$; however, it is encouraging that a recent study found minimal long-term effects of concussion on academic performance in high school at a population level. ${ }^{48} \mathrm{~A}$ range of strategies/accommodations to support RTS following concussion have been proposed, including temporary school absence. ${ }^{12-1635-37} 39$ In the initial few days following a concussion, students may need to stay home with relative restriction of physical and cognitive activities to allow acute symptoms to improve. Exacerbation of symptoms on RTS may indicate suboptimal accommodations or students pushing through symptoms. A secondary analysis of Thomas et al's study ${ }^{38}$ found that symptom spikes were associated with abrupt increases in mental activity, such as school attendance and extracurricular activities, in some patients but that most students were able to RTS without symptom exacerbation. ${ }^{11}$ This analysis found that symptom spikes were associated with higher symptom burdens and may be unrelated to physical or mental activity but rather other factors, such as stress or poor sleep. Conversely, some evidence indicates that longer restriction of activity, including school absence, may prolong recovery and increase symptoms. ${ }^{38}$ However, the optimal length of school absence has not been determined, and is likely to vary according to type, severity and duration of symptoms. Further study of when to recommend RTS is needed to guide clinical practice.
Many review articles and consensus guidelines for RTS recommend minimising school absence to avoid possible secondary problems such as social isolation, depression and anxiety about mounting schoolwork. ${ }^{12-19} 40$ 49-52 These guidelines also emphasise that students do not need to be symptom-free to go back to school, although this recommendation requires further validation. ${ }^{12-14} 1819{ }^{40}{ }^{49-52}$ Current clinically derived, consensus-based recommendations suggest that as symptoms improve and students are able to tolerate about $30 \mathrm{~min}$ of cognitive activity at home without significant symptom exacerbation, they can try RTS with accommodations as needed. Return to cognitive activity/ school may be facilitated by following a stepwise, symptom-limited programme, such as the strategy proposed in the most recent concussion consensus statement (table 3). ${ }^{1618194053-55}$ Generic RTS protocols may need to be adapted to different developmental/ school levels but more research is needed in this area. ${ }^{56}$

Provision/receipt of other academic accommodations may prevent worsening of symptoms on RTS. Identification of symptom profiles and screening for specific postconcussion deficits, such as visual disturbances and vestibulo-ocular deficits, can be useful in predicting students with higher academic needs requiring symptom-targeted interventions. ${ }^{31} 4143 \quad 45$ Specific academic accommodations, other than a period of school absence, were not examined in the reviewed studies but many guidelines, review articles and a survey of school nurses in Massachusetts list symptom-specific accommodations, ${ }^{12-16} 40$ 49-54 56 57 including:

- frequent breaks

- reduced workload, modified assignments

- no tests initially

- more time to complete assignments

- preferential seating in the classroom

- reduced visual and auditory stimulation.

Strategies specific to vision-related problems include avoidance of electronic screens, frequent visual breaks, preprinted notes in large font, audiobooks and oral teaching. ${ }^{435}$

To ensure students recovering from concussion are supported at school and receive accommodations as necessary, school personnel, parents and students need to be educated about current concussion management strategies. ${ }^{13} 40495658-61$ Surveys of teachers, school principals, athletic therapists, school nurses and parents have identified that concussion education, particularly of school staff and parents, is a key factor for successfully implementing an RTS protocol. ${ }^{565-62}$ An RCT demonstrated the effectiveness of a concussion policy as schools with an explicit policy were more likely to implement best practice guidelines for concussion management, and concussed students were more likely to receive academic accommodations after concussion. ${ }^{36} \mathrm{~A}$ case study also found that schools who receive concussion education provide more academic accommodations to students after concussion and had more resources available. ${ }^{58}$

\begin{tabular}{|c|c|c|}
\hline Mental activity & Activity at each step & Goal of each step \\
\hline $\begin{array}{l}\text { 1. Daily activities that do not give the child } \\
\text { symptoms }\end{array}$ & $\begin{array}{l}\text { Typical activities that the child does during the day as long as they do not } \\
\text { increase symptoms (eg, reading, texting, screen time). Start with 5-15 min at } \\
\text { a time and gradually build up }\end{array}$ & Gradual return to typical activities \\
\hline 2. School activities at home & Homework, reading or other cognitive activities outside of the classroom & Increase tolerance to cognitive work \\
\hline 3. Return to school part-time & $\begin{array}{l}\text { Gradual introduction of schoolwork. May need to start with a partial school } \\
\text { day or with increased breaks during the day }\end{array}$ & Increase academic activities \\
\hline 4. Return to school full-time & Gradually progress school activities until a full day can be tolerated & $\begin{array}{l}\text { Return to full academic activities and catch } \\
\text { up on missed work }\end{array}$ \\
\hline
\end{tabular}


Evidence-based education programmes and online educational tools can be effective to increase knowledge of concussion and management. ${ }^{366364}$

Development of school concussion policies can be guided by government legislation. The Ministry of Education in the province of Ontario, Canada, produced a policy memorandum mandating that all provincial school boards develop and implement a concussion policy ${ }^{65}$ This policy was evaluated a year after implementation in one Ontario school board by survey which found that $92 \%$ of schools have an RTP protocol and 77\% of schools have an RTS protocol; however, staff training and parent education were identified as areas needing more development. ${ }^{61}$ In the USA, the National Collaborative on Childhood Brain Injury recommended five areas of policy/procedural consideration to implement appropriate infrastructure to support RTS of students with concussions, including: (1) definition and training of the interdisciplinary school team; (2) professional development of school and medical communities; (3) identification, assessment and progress monitoring protocols; (4) a flexible set of intervention strategies to accommodate students' recovery needs; and (5) systematised protocols for active communication among medical, school and family team members. ${ }^{66}$

Furthermore, medical guidance can facilitate implementation of school supports for returning students. Providing explicit school-informed discharge instructions and an RTS letter after the initial concussion diagnosis in the ED resulted in significantly greater provision of academic accommodations than when not systematically recommended. ${ }^{39}$ The sample letter provided (online supplementary appendix A) is recommended to ensure direct communication between the medical provider and the school regarding the student's concussion symptoms and needs. In addition, regular medical follow-up after concussion is crucial to monitor recovery and facilitate RTS. Healthcare providers should identify risk factors/modifiers, such as age and symptomatology, that may prolong recovery and necessitate more intensive accommodations. ${ }^{18}{ }^{19}$ For instance, adolescents tend to be more symptomatic and more anxious about concussion, and may require more support on RTS. ${ }^{29} 3034$

Finally, recovery from concussion for students occurs in various arenas, including home, school, potential job and social/ sport. Effective communication between the medical team, school personnel, student and parents/caregivers is essential to ensure successful RTS for every concussed student. ${ }^{53} 56$ 59-62 66 Numerous surveys of various stakeholders in concussion management in students have highlighted the need for effective communication between all members of the care team. ${ }^{56}{ }^{59-62}$ This support network can relieve anxiety about missing school, allow students to focus on recovery and facilitate symptom improvement. ${ }^{13} 40495058$ It is important to emphasise that full symptom resolution, resulting in full school reintegration, must precede full return to sport and participation in high-risk contact training and gameplay. ${ }^{13} 141819$ However, early introduction of symptom-limited physical activity, supervised by a qualified health professional, is appropriate and may help facilitate RTS. ${ }^{18}$

While evidence is emerging, this review highlights the lack of research to guide RTS following SRC in children and adolescents. The studies identified had high risk of bias and study designs were mostly lower levels of evidence ( 3 or 4 ); there were only two RCTs. Many questions remain unanswered, such as the optimal length of school absence following SRC and what specific accommodations should be offered. There is an urgent need for high-quality research on SRC in children and adolescents, including RTS, to better inform management guidelines.

\section{Limitations}

RTS has been only a recent focus of concussion management and this study identified only 17 studies on this topic. The reviewed studies involved mostly high school and college students, many with mixed age cohorts (children, adolescents and adults). Despite the explosion of concussion research in the last decade, little research has been conducted in children 5-12 years of age. In addition, some studies included non-SRC and did not focus on RTS after concussion. Since we included only English language studies, these results may not be culturally generalisable and there may be language bias.

Other factors limiting generalisation of these review findings include study design, location and sample size. Level 1 evidence was not available as the majority of studies were retrospective and cross-sectional designs, with only two lower quality RCTs. Most papers had external validity and recall bias, particularly those conducted in hospital ED and specialty concussion clinics. Some studies had small sample sizes and used variable definitions of concussion and prolonged recovery. Finally, many of the studies examined school issues/outcomes at a time when there was little guidance on effective in-school management and support. With more informed guidance about RTS and provision of effective symptom-directed accommodations, RTS outcomes will likely improve.

For analysis of risk of bias, we employed the Downs and Black checklist, which was developed as a tool to be used in reviews of non-randomised studies. While we accept that the Downs and Black checklist has its limitations, and is not a perfect fit for this systematic review, it was deemed the best available for this study. An alternative risk of bias tool that would be more appropriate to the topic of RTS after paediatric concussion has yet to be developed.

\section{RECOMMENDATIONS}

A. Schools should have a concussion policy which includes concussion education for teachers, staff, students and parents; defines individuals within the school to direct the provision of individualised student supports; and includes a mechanism to implement and monitor appropriate academic accommodations to students recovering from SRC.

B. On diagnosis of concussion, students should be provided with a medical letter to facilitate the provision/receipt of necessary academic accommodations.

C. Students should have early, ongoing medical follow-up following an SRC to identify symptom targets, monitor recovery and aid with RTS, with regular communication between the medical provider, family and school.

D. Students may require temporary absence from school after concussion. Factors such as age/grade, types and severity of symptoms should be considered in determining the length of school absence. An individualised gradual RTS plan should be considered based on recovery trajectory.

E. Clinicians should screen for specific deficits, such as visual and vestibulo-ocular disturbances, that may affect RTS and require symptom-specific academic accommodations, as well as assess risk factors/modifiers that may prolong concussion recovery, requiring more intensive academic accommodations. In particular, adolescents may require more academic support.

F. Future high-quality research should be conducted to determine the criteria for length of absence from school, and appropriate, individualised RTS accommodations that should be provided for children and adolescents with typically recovering and prolonged SRC symptoms. 


\section{CONCLUSIONS}

Students should rest physically and cognitively initially following a concussion with a gradual increase in activities as symptoms allow. RTS following SRC requires cooperation and communication between healthcare providers, school personnel, parents/caregivers and the student to ensure that individualised accommodations and support are provided to facilitate optimal recovery.

\section{What is already known?}

- The effects of sport-related concussion (SRC) in children and the recovery challenges are different from adults.

- Adolescents may take longer to recover from SRC than adults and younger children.

- Return to school should be a priority in the management of children and adolescents following SRC.

\section{What are the new findings?}

- Factors such as symptomatology, age/school level, course load and rest after injury can affect return to school following SRC.

- Schools with a concussion policy tend to offer more academic accommodations to students recovering from SRC.

- Regular medical follow-up after concussion and provision of a return to school letter can help facilitate the provision of academic support.

- Many students require a brief absence from school and academic accommodations on return to school following an SRC to avoid significant exacerbation of concussion symptoms.

Acknowledgements The authors gratefully acknowledge Helen Baxter, Austin Health Library, with peer review of search strategy, and Margaret Sampson for assistance with drafting the search strategy.

Contributors All authors contributed to the development of the search strategy. LKP and GAD ran the searches independently and reviewed the results, selected the papers and compiled the data extraction tables as well as the risk of bias assessment. GAG identified additional pertinent studies for inclusion in the systematic review not identified by the search strategy and provided a template of the RTS letter in Appendix A. All authors contributed to the writing and critical review of the manuscript.

Funding This research received no specific grant from any funding agency in the public, commercial or not-for-profit sectors.

Competing interests LKP and GAD received travel expenses for the Berlin Concussion Conference in October 2016.

Provenance and peer review Not commissioned; externally peer reviewed.

(c) Article author(s) (or their employer(s) unless otherwise stated in the text of the article) 2019. All rights reserved. No commercial use is permitted unless otherwise expressly granted.

\section{REFERENCES}

1 Harris AW, Jones CA, Rowe BH, et al. A population-based study of sport and recreation-related head injuries treated in a Canadian health region. J Sci Med Sport 2012;15:298-304

2 Bakhos LL, Lockhart GR, Myers R, et al. Emergency department visits for concussion in young child athletes. Pediatrics 2010;126:e550-e556.

3 Meehan WP, Mannix R. Pediatric concussions in United States emergency departments in the years 2002 to 2006. J Pediatr 2010;157:889-93.

4 Centers for Disease Control and Prevention. Nonfatal traumatic brain injuries related to sports and recreation activities among persons aged $\leq 19$ years--United States, 2001-2009. MMWR Morb Mortal Wkly Rep 2011;60:1337-42.

5 Browne GJ, Lam LT. Concussive head injury in children and adolescents related to sports and other leisure physical activities. Br J Sports Med 2006;40:163-8.
6 Gessel LM, Fields SK, Collins CL, et al. Concussions among United States high school and collegiate athletes. J Athl Train 2007;42:495-503.

7 Emery CA, Meeuwisse WH, McAllister JR. Survey of sport participation and sport injury in Calgary and area high schools. Clin J Sport Med 2006;16:20-6.

8 Field M, Collins MW, Lovell MR, et al. Does age play a role in recovery from sportsrelated concussion? A comparison of high school and collegiate athletes. J Pediatr 2003;142:546-53.

$9 \operatorname{Sim}$ A, Terryberry-Spohr L, Wilson KR. Prolonged recovery of memory functioning after mild traumatic brain injury in adolescent athletes. J Neurosurg 2008;108:511-6.

10 Fazio VC, Lovell MR, Pardini JE, et al. The relation between post concussion symptoms and neurocognitive performance in concussed athletes. NeuroRehabilitation 2007;22:207-16.

11 Silverberg ND, Iverson GL, McCrea M, et al. Activity-related symptom exacerbations after pediatric concussion. JAMA Pediatr 2016;170:946-53.

12 Halstead ME, Walter KD. Council on Sports Medicine and Fitness. American academy of pediatrics. Clinical report--sport-related concussion in children and adolescents. Pediatrics 2010:126:597-615.

13 Halstead ME, McAvoy K, Devore CD, et al. Returning to learning following a concussion. Pediatrics 2013:132:948-57.

14 Purcell LK. Canadian Paediatric Society, Healthy Active Living and Sports Medicine Committee. Sport-related concussion: evaluation and management. Paediatr Child Health 2014;19:153-8.

15 Guidelines for diagnosing and managing pediatric concussion. 1st edn: Ontario Neurotrauma Foundation, 2014.

16 DeMatteo C, Stazyk K, Giglia L, et al. A balanced protocol for return to school for children and youth following concussive injury. Clin Pediatr 2015;54:783-92.

17 McCrory P, Meeuwisse W, Aubry M, et al. Consensus statement on concussion in sport--the 4th International Conference on Concussion in Sport held in Zurich, November 2012. Clin J Sport Med 2013;23:89-117.

18 McCrory P, Meeuwisse W, Dvořák J, et al. Consensus statement on concussion in sport-the 5th international conference on concussion in sport held in Berlin, October 2016. Br J Sports Med 2017:51:838-47.

19 Davis GA, Anderson V, Babl FE, et al. What is the difference in concussion management in children as compared with adults? A systematic review. Br J Sports Med 2017:51:1-12.

20 Meeuwisse WH, Schneider KJ, Dvorák J, et al. The Berlin 2016 process: a summary of methodology for the 5th International Consensus Conference on Concussion in Sport. Br J Sports Med 2017;51:873-6.

21 Downs SH, Black N, Brown N. The feasibility of creating a checklist for the assessment of the methodological quality both of randomised and nonrandomised studies of health care interventions. J Epidemiol Community Health 1998;52:377-84

22 OCEBM Levels of Evidence Working Group. "The Oxford 2011 Levels of Evidence". Oxford centre for evidence-based medicine. http://www.cebm.net/index.aspx?0=5653

23 Baker JG, Leddy JJ, Darling SR, et al. Factors associated with problems for adolescents returning to the classroom after sport-related concussion. Clin Pediatr 2015;54:961-8.

24 Brown NJ, Mannix RC, O'Brien MJ, et al. Effect of cognitive activity level on duration of post-concussion symptoms. Pediatrics 2014;133:e299-304.

25 Corwin DJ, Wiebe DJ, Zonfrillo MR, et al. Vestibular deficits following youth concussion. J Pediatr 2015;166:1221-5.

26 Darling SR, Leddy JJ, Baker JG, et al. Evaluation of the Zurich Guidelines and exercise testing for return to play in adolescents following concussion. Clin J Sport Med 2014; $24: 128-33$

27 Lovell MR, Collins MW, Iverson GL, et al. Recovery from mild concussion in high school athletes. J Neurosurg 2003;98:296-301

28 Makki AY, Leddy J, Hinds A, et al. School attendance and symptoms in adolescents after sport-related concussion. Glob Pediatr Health 2016;3:1-3.

29 Purcell L, Harvey J, Seabrook JA. Patterns of recovery following sport-related concussion in children and adolescents. Clin Pediatr 2016;55:452-8.

30 Ransom DM, Vaughan CG, Pratson L, et al. Academic effects of concussion in children and adolescents. Pediatrics 2015;135:1043-50.

31 Ransom DM, Burns AR, Youngstrom EA, et al. Applying an evidence-based assessment model to identify students at risk for perceived academic problems following concussion. J Int Neuropsychol Soc 2016;22:1038-49.

32 Taubman B, Rosen F, McHugh J, et al. The timing of cognitive and physical rest and recovery in concussion. J Child Neurol 2016;31:1555-60.

33 Zuckerman SL, Zalneraitis BH, Totten DJ, et al. Socioeconomic status and outcomes after sport-related concussion: a preliminary investigation. J Neurosurg Pediatr 2017:19:652-61.

34 Carson JD, Lawrence DW, Kraft SA, et al. Premature return to play and return to learn after a sport-related concussion: physician's chart review. Can Fam Physician 2014;60:e310-e12-5.

35 Corwin DJ, Zonfrillo MR, Master CL, et al. Characteristics of prolonged concussion recovery in a pediatric subspecialty referral population. J Pediatr 2014;165:1207-15.

36 Glang $A E$, Koester MC, Chesnutt JC, et al. The effectiveness of a web-based resource in improving postconcussion management in high schools. J Adolesc Health 2015;56:91-7 
37 Grubenhoff JA, Deakyne SJ, Comstock RD, et al. Outpatient follow-up and return to school after emergency department evaluation among children with persistent postconcussion symptoms. Brain Inj 2015;29:1186-91.

38 Thomas DG, Apps JN, Hoffmann RG, et al. Benefits of strict rest after acute concussion: a randomized controlled trial. Pediatrics 2015;135:213-23.

39 Zuckerbraun NS, Atabaki S, Collins MW, et al. Use of modified acute concussion evaluation tools in the emergency department. Pediatrics 2014;133:635-42.

40 Master CL, Gioia GA, Leddy JJ, et al. Importance of 'return-to-learn' in pediatric and adolescent concussion. Pediatr Ann 2012;41:1-6.

41 Swanson MW, Weise KK, Dreer LE, et al. Academic difficulty and vision symptoms in children with concussion. Optom Vis Sci 2017;94:60-7.

42 Ellis MJ, Cordingley DM, Vis S, et al. Clinical predictors of vestibulo-ocular dysfunction in pediatric sports-related concussion. J Neurosurg Pediatr 2017; 19:38-45.

43 Master CL, Scheiman M, Gallaway M, et al. Vision diagnoses are common after concussion in adolescents. Clin Pediatr 2016;55:260-7.

44 Ellis MJ, Cordingley D, Vis S, et al. Vestibulo-ocular dysfunction in pediatric sportsrelated concussion. J Neurosurg Pediatr 2015;16:248-55.

45 Pearce KL, Sufrinko A, Lau BC, et al. Near point of convergence after a sport-related concussion. Am J Sports Med 2015;43:3055-61.

46 Zemek R, Barrowman N, Freedman SB, et al. Clinical risk score for persistent postconcussion symptoms among children with acute concussion in the ED. JAMA 2016;315:1014-25.

47 Elbin RJ, Sufrinko A, Schatz P, et al. Removal from play after concussion and recovery time. Pediatrics 2016;138:e20160910.

48 Russell K, Hutchison MG, Selci E, et al. Academic outcomes in high-school students after a concussion: a retrospective population-based analysis. PLoS One 2016;11:e0165116.

49 McGrath N. Supporting the student-athlete's return to the classroom after a sportrelated concussion. J Athl Train 2010;45:492-8.

50 Sady MD, Vaughan CG, Gioia GA. School and the concussed youth: recommendations for concussion education and management. Phys Med Rehabil Clin N Am 2011;22:701-19.

51 Makdissi M, Davis G, Jordan B, et al. Revisiting the modifiers: how should the evaluation and management of acute concussions differ in specific groups? $\mathrm{Br} J$ Sports Med 2013;47:314-20.
52 Davis GA, Purcell LK. The evaluation and management of acute concussion differs in young children. Br J Sports Med 2014;48:1-5.

53 Gioia GA. Medical-school partnership in guiding return to school following mild traumatic brain injury in youth. J Child Neurol 2016;31:93-108.

54 Iverson GL, Gioia GA. Returning to school following sport-related concussion. Phys Med Rehabil Clin N Am 2016;27:429-36.

55 Davis GD, Purcell L, Schneider KJ, et al. Sport concussion assessment tool for childrens ages 5 to 12 years. Br J Sports Med 2017;51:862-9.

56 Blackwell LS, Robinson RF, Proctor MR, et al. Same care, different populations: returnto-learn practices following concussion in primary and secondary schools. J Child Neurol 2017:32:327-33.

57 Grady MF, Master CL. Return to school and learning after concussion: tips for pediatricians. Pediatr Ann 2017;46:e93-e98.

58 Davies SC, Sandlund JM, Lopez LB. School-based consultation to improve concussion recognition and response. J Educ Psychol Consult 2016:26:49-62.

59 Wing R, Amanullah S, Jacobs E, et al. Heads up: communication is key in school nurses' preparedness for facilitating "return to learn" following concussion. Clin Pediatr 2016;55:228-35.

60 Lyons VH, Moore M, Guiney R, et al. Strategies to address unmet needs and facilitate return to learn guideline adoption following concussion. J Sch Health 2017;87:416-26

61 Hachem LD, Kourtis G, Mylabathula S, et al. Experience with Canada's first policy on concussion education and management in schools. Can J Neurol Sci 2016;43:554-60

62 Kasamatsu T, Cleary M, Bennett J, et al. Examining academic support after concussion for the adolescent student-athlete: perspectives of the athletic trainer. J Ath/ Train 2016;51:153-61.

63 Graff DM, Caperell KS. Concussion management in the classroom. J Child Neurol 2016:31:1569-74.

64 Hunt AW, De Feo L, Macintyre J, et al. Development and feasibility of an evidenceinformed self-management education program in pediatric concussion rehabilitation. BMC Health Serv Res 2016;16:400.

65 Ontario Ministry of Education. Policy/program memorandum no. 158: school board policies on concussion, 2014

66 Gioia GA, Glang AE, Hooper SR, et al. Building statewide infrastructure for the academic support of students with mild traumatic brain injury. J Head Trauma Rehabil 2016;31:397-406. 